\title{
Mejora de la calidad de vida en pacientes con baja adherencia al tratamiento. Intervenciones en dependientes del alcohol "Sin Hogar"
}

\author{
Pedro Cuadrado Callejo \\ Psiquiatra. Coordinador del Programa de Problemas Relacionados con el Alcohol del Servicio de Salud Mental Retiro. \\ Servicio de Salud Mental Retiro. Madrid. Consejería de Sanidad. Comunidad de Madrid \\ Pedro Cuadrado Callejo. SSM Vallecas Villa. C/ San Claudio 154. 28038 Madrid. Tfno. 913909953. Correo electrónico: pedrocuadrado@mi.madritel.es
}

\section{RESUMEN}

Objetivo. Revisar bibliografía sobre dependientes del alcohol sin hogar, su prevalencia, características y programas de intervención. Proponer un modelo de atención para los mismos. Resultados. Entre un 25 y un $45 \%$ de las personas sin hogar tienen un trastorno por alcohol. Son más jóvenes, de comienzo más temprano, mayor consumo y mayor duración de la dependencia y más complicaciones y comorbilidad psiquiátrica que los dependientes de alcohol con residencia. Los programas asistenciales existentes no son eficaces y más del $75 \%$ no reciben ningún tratamiento. Propuesta de intervención. Los objetivos básicos deben ser conseguir el enganche y la retención en el tratamiento y la actuación sobre necesidades básicas y alojamiento. Se van a precisar técnicas específicas, estrategias de intervención en la calle y monitorización intensiva que favorezca el acceso a los dispositivos asistenciales y el cumplimiento del tratamiento. Conclusiones. Los dependientes del alcohol sin hogar tienen unas necesidades asistenciales complejas cuyo abordaje requiere programas específicos que se deben apoyar en la coordinación y adaptación de los servicios que intervienen, la discriminación positiva de estos pacientes en los recursos de la red, la creación de equipos multidisciplinares de trabajo en la calle, recursos de alojamiento y ayudas económicas.

Palabras clave: Dependencia alcohólica. Alcoholismo. Personas Sin Hogar.

\section{INTRODUCCIÓN}

0 i analizamos lo que ha pasado con los dependientes del alcohol al cabo de varios años de iniciar tratamiento, nos encontramos con tres grupos de pacientes. Aquellos que completaron el tratamiento y que fueron dados de alta, que suponen entre el 10 y el $15 \%$; los que no completan el programa de tratamiento, que son entre la mitad y el 60\%; y aquellos que por su evolución permanecen en los programas de tratamiento durante años de forma prolongada y que suponen un tercio de la muestra ${ }^{11,2)}$. Cuando se estudian las características de estos grupos ${ }^{(1)}$, se encuentra que los que evolucionan mal y

\section{ABSTRACT}

Objective. Review the bibliography on homeless alcohol dependents, prevalence, characteristics and intervention programmes and propose a treatment model for them. Results. Between 25 and $45 \%$ of the homeless have some alcohol disorder. They are younger, start earlier, drink more and their dependence lasts longer. They present more complications and psychiatric comorbidity than alcohol dependents who do have a home. The existing assistance programmes are not effective and more than $75 \%$ do not receive any treatment. Intervention proposal. Specific objectives must encourage them to seek treatment and to continue it, meet their basic needs and provide accommodation. Specific techniques will be needed as well as street intervention strategies and intensive monitoring which promotes access to available treatment and the means to continue with it. Conclusions. Alcohol dependent homeless have complex welfare needs which must be met by specific programmes backed by the coordination and adaptation of the services involved, positive discrimination towards those patients in every welfare resource, the creation of specific multidisciplinary teams working on the street, accommodation resources and financial assistance.

Key words: Alcohol Dependence. Alcoholism. Homeless. Treatment.

permanecen en tratamiento tienen más deterioro y más psicopatología asociada así como más conciencia de enfermedad y, sobre todo, un alto soporte sociofamiliar; mientras que los que abandonan el tratamiento presentan menos deterioro neuropsicológico y menos psicopatología asociada pero, también, menos conciencia de enfermedad y menos implicación y soporte socio-familiar.

A este último grupo, de no cumplidores del tratamiento, hay que añadir otro constituido por aquellos que, pueden suponer un $5 \%$ de todos los dependientes del alcohol ${ }^{(3)}$, y que se caracterizan por no aparecer por los dispositivos asistenciales normalizados, o 
hacerlo de una manera puntual, y por compartir características de los que abandonan y de los que se cronifican: con múltiples y severas complicaciones y altos niveles de psicopatología asociada, pero que no tienen compromiso con el tratamiento y carecen del mínimo soporte socio-familiar ${ }^{(4)}$, y que forman parte de un grupo más amplio que son los Enfermos Mentales Sin Hogar que constituyen casi la mitad de un colectivo muy heterogeneo agrupado en torno al término de Personas Sin Hogar.

Desde el punto de vista sociológico, Persona Sin Hogar es un término complejo que puede hacer referencia a distintas situaciones. El Consejo de Europa en 1992 definía los criterios para su consideración ${ }^{(5)}$ : 1) No tener techo sobre su cabeza, estando condenado a vivir en la calle como vagabundo, 2) estar temporalmente alojado en refugios o centros de acogida especialmente creados para personas sin techo por las autoridades públicas o por el sector privado, 3) está temporalmente alojado en el sector privado, en establecimientos de cama y desayuno, hoteles baratos $u$ otros albergues privados, o con amigos o familiares con los que se ven forzados a cohabitar, 4) ocupar legal o ilegalmente viviendas inseguras, cabañas, chozas, casa abandonadas, barracas, etc., 5) residir en instalaciones, establecimientos de cuidados infantiles, hospitales, prisiones y hospitales psiquiátricos y que no tienen domicilio al que ir al salir de las mismas, 6) tener una vivienda que no se puede considerar adecuada o socialmente aceptable y que, por tanto, se convierten en personas o familias mal alojadas.

La situación de Sin Hogar en los enfermos mentales es el resultado de la imbricación de factores individuales, ligados a la propia patología, y factores psico-sociales y económicos. Tiene que ver con situaciones de desarraigo familiar y social, desempleo, pobreza severa, deterioro personal y social para los que los recursos existentes (sociales y sanitarios) no logran aportar respuestas. En este sentido, la escasez de recursos intermedios, residenciales y de rehabilitación, el desarrollo comunitario insuficiente y la escasa implantación de modos de trabajo más flexibles y ligados a la calle; puede considerarse uno de los elementos claves que explican el fracaso en la prevención de la exclusión en los enfermos mentales ${ }^{(6)}$.

El objetivo de este trabajo es analizar el grupo de dependientes del alcohol integrados dentro del colectivo de Enfermos Mentales Sin Hogar: su prevalencia, características clínicas, utilización de recursos y programas de intervención así como proponer un modelo de atención que se adapte a sus características y permita cubrir sus necesidades asistenciales.

\section{DIMENSION DEL PROBLEMA}

Los estudio epidemiológicos llevados a cabo en distintos paises europeos, sitúan las cifras de Perso- nas Sin Hogar en torno a 7.5 por mil habitantes ${ }^{(7)}$. En España estaríamos un poco por debajo de la media europea, alrededor del 7 por mil ${ }^{(5)}$.

Dentro de los muchos problemas que presenta esta población, la patología psiquiátrica es una de las más prevalentes. Aunque las cifras varían mucho de unos estudios a otros, sobre todo por la selección de la muestra, se estima que entre un tercio y la mitad de las Personas Sin Hogar sufren algún trastorno mental $(8,9,10,11,12,5)$, el doble de lo que se encuentra en la población domiciliada, siendo los trastornos por alcohol la patología más frecuente.

La definición de Persona Sin Hogar, el lugar de selección de la muestra y los criterios para los trastornos por alcohol, van a determinar las tasas de prevalencia, con cifras que van desde el $23 \%$ al $63 \%{ }^{113,14,15,}$ $10,16,17,18)$. Estas cifras suponen que la presencia de trastonos por alcohol en esta población es de dos veces y media a cinco veces más frecuente que en la población general ${ }^{(4,12)}$.

En España (TABLA 1), las tasas de trastornos por alcohol en Personas Sin Hogar, van desde el $24 \%$ encontrado por Vega ${ }^{(19)}$, en Gijón, en una muestra recogida en una Oficina de Información para Personas Sin Hogar, cercana al 29\% encontrado Por Rico y col. (20) en el Albergue de Aranjuez, hasta el 48\% de Lucas y Col. ${ }^{(21)}$, en Barcelona, en una muestra recogida en la calle y el $44 \%$ encontrado por Muñoz y col. ${ }^{(5)}$, en Madrid, en una muestra recogida en distintos ámbitos donde se encuentran los Sin Hogar.

\section{TABLA 1. Trastornos por alcohol en muestras de Sin Hogar}

\begin{tabular}{|lccc|}
\hline AUTORES & $\begin{array}{c}\text { Origen } \\
\text { Muestra }\end{array}$ & N & $\begin{array}{c}\text { T. por Alcohol } \\
\text { (DSM III-R) }\end{array}$ \\
\hline $\begin{array}{l}\text { Rico, Vega y Aranguren } \\
\text { (Aranjuez 1994) }\end{array}$ & (Albergue) & 524 & $29 \%$ \\
$\begin{array}{l}\text { Lucas y col. } \\
\text { (Barcelona 1995) }\end{array}$ & (Calle) & 99 & $48 \%$ \\
$\begin{array}{l}\text { Vega } \\
\text { (Gijón 1996) }\end{array}$ & $\begin{array}{l}\text { (Oficina de Infor. } \\
\text { De Sin Hogar) }\end{array}$ & 170 & $24 \%$ \\
$\begin{array}{l}\text { Muñoz, Vargas y Cruzado } \\
\text { (Madrid 1999) }\end{array}$ & $\begin{array}{c}\text { (Albergues, } \\
\text { Comedores, calle) }\end{array}$ & 262 & $44 \%$ \\
\hline
\end{tabular}

\section{CARACTERISTICAS DE LOS DEPENDIENTES DEL ALCOHOL SIN HOGAR}

Wright y Devine ${ }^{(22)}$, en un estudio sobre 8000 homeless de Nueva Orleans, 3/4 de los cuales tenían un trastorno por alcohol, no encuentran muchas diferencias entre aquellos con y sin trastornos por alcohol asociados; aunque algunos trabajos si encuentran una 
mayor duración de la situación de Sin Hogar ${ }^{(10,20)}$. Sin embargo si existen diferencias importantes cuando se comparan con los dependientes del alcohol con estabilidad residencial; a pesar de que la mayoría de los dependientes del alcohol Sin Hogar cumplen los criterios de un trastorno por alcohol antes de llegar a esta situación ${ }^{(23)}$.

Koegel y Burnam ${ }^{(4)}$, en uno de los primeros estudios de Sin Hogar centrado en los trastornos por alcohol (abuso/dependencia), tomando los datos del estudio ECA de Los Angeles, compararon el grupo de trastornos por alcohol en personas Sin Hogar con aquellos con estabilidad residencial, encontrando que los Sin Hogar eran más jóvenes, con más frecuencia no habían estado casados y si lo estuvieron más separados y divorciados, menos probabilidad de haber tenido hijos, no habían diferencias en cuanto al sexo y el nivel educacional, tenían más antecedentes de dificultades y problemas de conducta en la infancia, comienzo más temprano y mayor duración de la dependencia, patrón estable o continuo con mayores cantidades de alcohol consumido, más blackouts y más frecuentes y mas graves complicaciones somáticas en general, más violencia y mayores complicaciones legales, familiares y socio-laborales y más trastornos psiquiátricos asociados, vez y medía más comorbilidad que en los trastornos por alcohol con residencia. Entre los trastornos psiquiátricos asociados cabe resaltar el gran incremento de la presencia de esquizofrenia (8.7 veces más que en aquellos con residencia), deterioro y demencia (6.3 veces), trastornos afectivos (2.2 veces) y abuso de otras drogas (1.4 veces). Muñoz y col. (24), en un estudio similar, comparan los datos de Los Angeles con una muestra de Madrid, encontrando pocas diferencias excepto un mayor consumo de cocaína en Los Angeles y de alcohol en Madrid. Otros estudios, en otras muestras, han confirmado una buena parte de estos datos.

La tasa de trastornos por alcohol en la población Sin Hogar tiende a igualarse en cuanto al sexo: un 10\% de todos los dependientes del alcohol Sin Hogar son mujeres ${ }^{(3,14)}$, tasa que se corresponde a la de mujeres en el total de la población Sin Hogar ${ }^{(12,5)}$. Un hallazgo general es el de un comienzo más precoz, mayor duración de la dependencia, mayores cantidades de alcohol consumido y más complicaciones asociadas ${ }^{(14,25,10)}$, con alrededor de la mitad con antecedentes de haber estado en prisión ${ }^{(26,14)}$ y y más de dos tercios, casi el doble que en aquellos con residencia, de comorbilidad psiquiátrica ${ }^{27}$, 15, 28), con alta asociación a trastornos por consumo de otras drogas ${ }^{(14,28)}$, así como más historia familiar de alcoholismo ${ }^{(26)}$.

\subsection{UTILIZACIÓN DE RECURSOS ASISTENCIALES Y RESULTADOS}

En cuanto a la búsqueda de tratamiento, la gran mayoría de los dependientes del alcohol Sin Hogar nunca han estado en tratamiento. Así, Rico y col. ${ }^{(20)}$, en su muestra de Sin Hogar del albergue de Aranjuez, encuentran que un $84 \%$ no han recibido nunca tratamiento para su trastorno por alcohol, el $8 \%$ lo habían recibido antes de ser transeuntes, el 5\% siendo transeuntes y un $3 \%$ seguían tratamiento por dicha patología. En otras muestras, recogidas en ámbitos no asistenciales, los resultados son similares: Gelberg y col. ${ }^{(10)}$, en Los Angeles, encuentra que un $78 \%$ no han estado en tratamiento y un $75 \%$ encuentran Wright y Devine ${ }^{221}$, en su muestra de 8000 Sin Hogar de Nueva Orleans. Además; por sus características clínicas y sociales, su pobre cumplimiento y los malos resultados obtenidos; una parte de estos pacientes no son admitidos en los dispositivos normalizados. Así Nuttbrock y col. ${ }^{(29)}$, en New York, encuentran que alrededor de un tercio son rechazados que, unido a otro tercio que rechazan los programas que se les ofrecen, reduce a un tercio la población tratada de la que acude a los dispositivos asistenciales. Sin embargo, aquellos que se tratan utilizan de forma intensiva los programas de tratamiento. Así, se encuentra que entre la mitad y dos tercios de los que acuden a programas de tratamiento tienen antecedentes de tratamientos previos ${ }^{(4,14)}$, más de la mitad han ingresado para desintoxicación ${ }^{(4)}$, y lo han hecho varias veces, con una media de cinco ingresos previos y un $11 \%$ lo han hecho más de diez veces ${ }^{(14)}$.

En cuanto a los resultados del tratamiento, la mayoría de los factores que influyen en la buena evolución como son el refuerzo comunitario, menores cambios de vida y de residencia, el empleo regular, el estar casado o con pareja, el vivir acompañado y el mayor status socioeconómico, asi como la denominada coerción no legal (miedo a perder familia, trabajo, salud, posición social) (30), están ausentes en el grupo de dependientes del alcohol Sin Hogar. Estos datos se confirman en los pocos estudios que hay sobre resultados del tratamiento. Así, Feuerlein (31), tomando datos de un estudio multicéntrico alemán, sobre 1410 pacientes ingresados con posterior seguimiento hasta los 18 meses, encuentra que las tasas de abstinencia en esta población es entre seis y siete veces peor que en aquellos con residencia, y mayores tasas de abandono en el seguimiento ( $9 \%$ abstinentes frente a $57 \%)$. Los resultados, en los programas específicos, van a depender del cumplimiento y permanecia en el tratamiento ${ }^{(22,29)}$, y aunque algunos estudios no encuentran diferencias, con los dependientes del alcohol con residencia, en seguimientos a corto plazo ${ }^{(25)}$, en otros trabajos, el cumplimiento, es muy bajo, alrededor de un tercio de los pacientes que lo inician lo completan y permanecen, como media, menos de un tercio del tiempo de duración del programa ${ }^{(22,29)}$.

Habría por lo tanto tres grandes grupos de dependientes de alcohol Sin Hogar, aquellos que se integran sin dificultad en los programas asistenciales pero que 
por su mala evolución los utilizan de manera repetida, sobre todo las unidades de desintoxicación e internamiento, y, además, prolongan sus estancias en los mismos; un segundo grupo que no se adapta a los dispositivos asistenciales existentes y son rechazados por éstos o el paciente rechaza incorporarse a los mismos; y un tercer grupo, seguramente el más numeroso, que no ha acudido ni acude a los dispositivos asistenciales y, si lo hace, no es para resolver sus problemas de alcohol.

\section{PROGRAMAS DE INTERVENCION}

\subsection{PRINCIPIOS GENERALES}

Los programas dirigidos a los dependientes del alcohol Sin Hogar comparten barreras, dificultades, objetivos y estrategías con un conjunto de intervenciones, que se han ido desarrollando a partir de la década de los ochenta, para lo que se ha denominado patología dual. Esto es así por que una gran parte de los pacientes con patología dual son también Personas Sin Hogar ${ }^{(32)}$.

La principal barrera para el tratamiento es la falta de residencia y de redes sociales mínimas, de ahí que las intervenciones sociales y el problema del alojamiento sean prerrequisitos a cualquier actuación ${ }^{27,33)}$. La falta de búsqueda de tratamiento y de adaptación a las características de los dispositivos asistenciales normalizados, hacen que el enganche, primera fase del tratamiento, se convierte en un elemento primordial del programa. Para su consecución, se van a utilizar distintas estrategias, como son las intervenciones fuera de los centros sanitarios: allí donde ellos viven, y pasan el tiempo; así como técnicas específicas, como intervenciones motivacionales, de consejo y de apoyo social ${ }^{(33)}$. Además, se va a precisar una monitorizacion estrecha e intensiva tanto para resolver las situaciones agudas de su enfermedad, con internamientos repetidos y prolongados ${ }^{(34)}$, como en el tratamiento de mantenimiento a lo largo del tiempo, lo que puede requerir de dispositivos como hospitales de día, pisos supervisados o unidades residenciales, aunque algunos trabajos han puesto de manifiesto que una buena parte de estos pacientes no se benefician de tratamientos residenciales intensivos ${ }^{(35,36)}$.

Un único equipo multidisciplinar, flexible y con preparación y especialización adecuada, se hará cargo del tratamiento integral. Serán "equipos de tratamiento continuo" que tomen la responsabilidad total de un pequeño grupo de pacientes, con objetivos básicos de enganche y estabilización ${ }^{(37)}$. Algunos trabajos han demostrado claramente cómo, la integración de intervenciones sobre el abusos de sustancias, los problemas de salud mental asociados y los problemas residenciales y de apoyo social; se traduce en menos días de ingreso y más días de vivienda estable, mejoría del trastorno por alcohol y drogas, mejoría de los síntomas psiquiátricos, estatus funcional y calidad de vida ${ }^{(38)}$, y tasas mas bajas de abandono ${ }^{(39,28)}$.

Por último, un aspecto importante en el cumplimiento de objetivos y en el éxito de estos programas es la actitud de los profesionales y de los equipos que se debe guiar por el optimismo, la sensibilidad hacia otras culturas, una buena parte de estos pacientes son inmigrantes, la competencia y especialización profesional, el trabajo de motivación sobre los pacientes y una perspectiva longitudinal, planteando el tratamiento como un camino largo donde los resultados no se ven a corto plazo ${ }^{(33)}$.

\subsection{MODELOS DE INTERVENCION}

Las dificultades de encaje en los sistemas sanitarios normalizados, han hecho que la población Sin Hogar con trastornos psiquiátricos asociados y, como parte de ellos, los dependientes del alcohol hayan estado, en la mayoría de los sitios, a expensas de soluciones puntuales dependientes de organismos públicos, fundamentalmente municipios, y de otros colectivos sociales implicados en funciones de beneficencia, con un perfil y un carácter fundamentalmente voluntarista. A medida que el problema iba creciendo en magnitud y las repercusiones sobre otros colectivos sociales (aumento de la inseguridad, violencia, mala imagen), se han ido incrementando, esas soluciones puntuales se han consolidado. En este momento, se pueden diferenciar dos grandes modelos de intervención: uno apoyado en una red de servicios específicos, implantado sobre todo en USA ${ }^{139,14 .}$ ${ }^{221}$, y otro que gira alrededor de programas específicos integrados en dispositivos de toxicomanías ${ }^{(26,40,32)}$.

\subsubsection{Modelo de dispositivos específicos}

El National Institute on Alcohol Abuse and Alcoholism (NIAAA) editó en 1988 un libró titulado "Alcohol Recovery Programs for Homeless people. A survey of Current Programs In the U.S." ${ }^{(39)}$, donde se describen 15 programas asistenciales para problemas de alcohol en personas sin hogar en USA.

En todos ellos se diferencian tres componentes, aunque cada programa hace más hincapié en unos que en otros: servicios de ingreso, servicios de recuperación temprana y servicios de mantenimiento de la recuperación. Los Servicios de ingreso tienen como función atraer a los paciente, utilizándose diferentes estrategias como son la búsqueda activa, por las calles con patrullas móviles para los intoxicados, búsqueda pasiva en los lugares donde paran los Sin 
Hogar y protección y custodia, con traslado a los intoxicados a los centros de desintoxicación o lugares de sobriedad. El Servicio de Recuperación Temprana tiene como objetivos el conseguir la abstinencia y establecer la sobriedad y se hace a través de unidades de desintoxicación de alrededor de cinco días de duración y distintas alternativas residenciales durante un periodo de tres a seis meses para permitir la recuperación física y mental de los pacientes, con vuelta a empezar si hay recaída. Los Servicios de Mantenimiento de la Recuperación, funcionan como servicios de apoyo y seguimiento que tratan de cubrir las necesidades básicas de los pacientes.

Los autores de este trabajo, extraen una serie de conclusiones, de las que señalamos aquellas que vienen a definir la esencia y los puntos débiles de los programas llevados a cabo en los dispositivos específicos: 1) Ausencia de política pública de asistencia a las personas con problemas de alcohol sin hogar. No hay políticas locales consistentes y no existe una política nacional para la prevención y tratamiento de los problemas de alcohol entre los Sin Hogar, manejándose esta población como el resto de los Sin Hogar. 2) Los Sin Hogar con problemas de alcohol son un grupo muy diverso, encontrándose personas cada vez más jóvenes, más minorías, más mujeres, más poliabuso de drogas, más enfermos mentales, con menor educación y menos habilidades vocacionales que necesitarían programas adaptados a sus peculiaridades. 3) Son programas desarrolladas por iniciativas personales y con una características ligadas a las características de las personas que los ponen en marcha, y el origen y la orientación de los lideres de los programas es muy diversa, pudiendo estar compuestos tanto por profesionales como por pacientes recuperados . 4) La coordinación con los servicios de salud mental y de alcohol suele estar ausente. Algunos programas de alcohol con una mentalidad AA son reaccios a admitir la intervención de los servicios de salud mental. 5) Es imprescindible conectar los servicios de alcohol con otras agencias y servicios para poder cubrir las necesidades de estas personas de una manera integral.

\subsubsection{Modelo de programas específicos inte- grados en dispositivos de toxicomanías.}

En el mundo anglosajón, estos programas vienen de la mano de la asistencia a la patología dual. Un buen ejemplo es el programa que se crea, a finales de la decada de los setenta, en el Bellevue Hospital de New York, que consta de tres dispositivos independientes pero coordinados entre si, una Sala de Internamiento y un Centro de Día para patología dual donde casi la mitad eran Sin Hogar y una Casa a Medio Camino donde casi el 90\% eran Sin Hogar ${ }^{132)}$.

En España, surgen en la década de los ochenta, sobre todo en las grandes ciudades como Barcelona y
Madrid, y lo hacen ligados a los programas municipales de toxicomanías y este origen va a condicionar su implantación y desarrollo. El de Barcelona, se crea como un equipo multidisciplinar con funciones de desintoxicación, terapia individual y grupal, y atención social. A los diez años, su implantación era escasa, con carencias importantes y con la constatación de que la mayoría de los recursos asistenciales iban dirigidos a aquellas personas Sin Hogar con problemas de drogas ilegales ${ }^{(26)}$. En Madrid, funciona un programa de Atención a Drogodependientes Sin Hogar, dependiente del Ayuntamiento de Madrid y coordinado con los Centros de Atención a Drogodependientes (CAD), con un buen nivel de desarrollo (39); pero donde tienen escasa cabida los problemas de alcohol, que son atendidos en un Centro de Alcoholismo y Tabaquismo a través de un programa ambulatorio normalizado, sin recursos de internamiento y residenciales, con una baja implicación de los servicios sociales y sin coordinación con el sistema sanitario general que es donde se atiende, en Madrid, a los problemas de alcohol de las personas con residencia.

Los programas de Madrid y Barcelona ejemplifican la atención que se presta a los problemas de alcohol, por parte del Plan Nacional sobre Drogas. Dentro de la Estrategia Nacional sobre Drogas aprobada en 1999 (41), y donde se fijan objetivos y actuaciones a conseguir entre el 2003 y el 2008, una de las Areas de Intervención que se contemplan es la de Reducción de Daños. Sin embargo, en el caso del alcohol, estos programas no se centran en los dependientes del alcohol sino que se contemplan, axclusivamente, en relación con los accidentes de tráfico y la violencia. Esto deja a los dependientes del alcohol Sin Hogar sin atención específica dentro de los programas de Toxicomanías, lo que unido a las necesidades de atención prioritaria a la patología médica y psiquiátrica asociada, es aconsejable incluir la atención a este colectivo dentro de la atención a la Salud Mental, en general, y en los programas de atención a Enfermos Mentales Sin Hogar, en particular.

\section{PROGRAMA ESPECIFICO PARA ENFERMOS MENTALES SIN HOGAR INTEGRADO EN DIS- POSITIVOS GENERALES}

En Europa, en los últimos años y promovido por el Consejo Regional Europeo de la Federación Mundial de Salud Mental, se ha puesto en marcha el proyecto Salud Mental Exclusión Social (SMES), que pretende llevar a cabo intervenciones coordinadas desde los profesionales sociales y sanitarios, públicos y privados, con el objetivo de mejorar la situación asistencial y las condiciones de vida, de los enfermos mentales en situación de exclusión social; así como promover el 
análisis y evaluación de la situación de este colectivo y de sus condicionantes. En España, un grupo incluido en este proyecto, Grupo SMES MADRID, ha hecho una propuesta de actuación sobre Enfermos Mentales Sin Hogargeneral ${ }^{(6)}$, con dos objetivos diferenciados: a) cubrir necesidades básicas expresadas por los propios pacientes y b) reintegrarlos a dispositivos normalizados de atención médica tanto para sus problemas médicos como psiquiatricos. Esta propuesta ha sido asumida, hace unos meses, por la administración autonómica que ha aprobado recientemente un plan específico de actuación sobre las Personas Sin Hogar con trastornos mentales.

Siguiendo las líneas marcadas por el Grupo SMES MADRID, la atención a los dependientes del alcohol Sin Hogar, se podría llevar a cabo a través de programas específicos integrados en los dispositivos sanitarios y de salud mental generales y con una coordinación estrecha con programas sociales específicos integrados en los servicios sociales generales.

\subsection{PROGRAMA SMES MADRID ADAPTADO A DEPENDIENTES DEL ALCOHOL}

El programa trataría de dar respuesta a un colectivo en el que se diferencian tres perfiles con necesidades asistenciales distintas: 1) Dependientes del alcohol que viven en la calle sin tratamiento y sin inserción en los servicios de salud mental, cuyas necesidades sanitarias (enganche, tratamiento y seguimiento) van a requerir equipos específicos para su aplicación. 2) Dependientes del alcohol que residen en centros de acogida, pensiones, etc., que utilizan distintos recursos de la red de atención a personas sin hogar y que una buena parte ya tienen tratamiento de salud mental. Sus necesidades sanitarias, inicialmente, pueden requerir de equipos específicos; siendo cubiertas, posteriormente, por los equipos de los programas de alcohol de los servicios de salud mental. 3) Dependientes del alcohol con dificultades de alojamiento, en condiciones psico-sociales precarias y en proceso de exclusión social. Es el grupo más amplio y suelen estar en contacto irregular con los servicios de salud mental y utilizan algunos de los recursos de la red de atención a personas sin hogar (comedores, talleres de inserción, centros de encuentro). Su atención se llevará a cabo por los equipos de los programas de alcohol de los servicios de salud mental.

En el programa se pueden diferenciar varios componentes: detección y enganche, evaluación, provisión de servicios básicos y tratamiento y seguimiento, que van a a ser desarrollados por distintos dispositivos y servicios y tener diferente peso y características en función del grupo de pacientes a quien va dirigido.

\subsubsection{Detección y engache.}

La detección, es el primer estadio y un componente básico en el grupo 1 (pacientes sin inserción en los servicios de salud mental), y se llevaría a cabo por los Servicios Sociales generales y especializados (Unidad Movil de Emergencia Social y Servicio de Información Telefónica y Atención de Emergencias Sociales) y otras organizaciones como albergues y Organizaciones no Gubernamentales implicadas en el cuidado de estas personas). Tras la detección emitirán un informe al equipo específico, que es el que va llevar a cabo el enganche y las distintas intervenciones.

El enganche es uno de los momentos claves del proceso. Se trata de ganar su confianza para que acepte ayuda $y$, si es posible, tratamiento. La manera de hacerlo es a través de un modelo de "búsqueda activa" (outreach): ir donde están, conocer lo que necesitan $y$, tras un largo proceso de contactos, ayudarles a conseguirlo y persuadirles para que acepten recibir ayuda. El trabajo en la calle, continuado y con un conocimiento individualizado de cada uno de ellos es indispensable. Sólo en casos excepcionales, de situaciones de grave riesgo o en aquellos en los que, por su psicopatología, han fracasado todos los intentos de iniciar un programa de tratamiento, puede tener sentido solicitar autorización judicial para hacerlo de forma involuntaria.

En el grupo $1 \mathrm{y}$, ocasional e inialmente, en el grupo 2 las funciones de enganche las debe llevar a cabo un equipo específico, que se ha denominado Equipo Multidisciplinar de Enganche y Seguimiento de Enfermos Mentales Sin Hogar ${ }^{(6)}$. Este equipo, en el grupo 1, cumple además todas las funciones de intervención y seguimiento a largo plazo, con los siguientes objetivos:evaluación psiquiátrica y social, valoración de la situación de urgencia y gestión de las actuaciones derivadas, lograr el enganche, garantizar las necesidades básicas en niveles de exigencia progresiva (alojamiento, alimentación, atención sanitaria), tutoría asertiva, y conectarlo a los servicios sanitarios y sociales con el fin de llevar a cabo un plan individualizado de tratamiento, seguimiento y rehabilitación.

\subsubsection{Evaluación.}

A todos se les propondrá una evaluación por parte de los programas de alcohol de los servicios de salud mental y en aquellos casos en los que no acepten, la realizarán los equipos específicos en los lugares donde los pacientes se encuentren. La evaluación será la base de un plan individualizado de actuación, que se intentar consensuar con el paciente, y que se puede llevar a cabo en el ámbito ambulatorio o iniciarse en regimen de hospitalización. Si el paciente no acepta el plan propuesto, pero necesita atención urgente y su situación clínica lo permite, se puede 
recurrir a la intervención judicial. En caso contrario, se sigue trabajando el enganche en la calle o en los lugares donde se encuentre.

\subsubsection{Provisión de servicios básicos.}

El nucleo de la atención lo va a constituir: 1) El Alojamiento, con utilización de distintos dispositivos según el grado de autonomía (Centros de Acogida, Centro de Atención Permanente a Enfermos Mentales Sin Hogar, Minirresidencia, Residencia asistida, Pisos Tutelados, Pensiones Supervisadas, Unidades de Rehabilitación); alimentación y ropa. 2) Ayudas económicas. 3) Defensa legal (incapacidad si procede, comisión de tutela). 4) Integracion laboral, facilitando la incorporación a programas de formación y empleo normalizados o específicos para drogodependientes.

5) Cuidados médicos y de salud mental.

La utilización de estrategias de "case management" o seguimiento de casos ${ }^{(42)}$, va a permitir la coordinación de dispositivos, profesionales y actuaciones necesarios para llevar a cabo esta atención.

\subsubsection{Tratamiento y seguimiento.}

Un grupo de pacientes se pueden incorporar al programa de tratamiento normalizado, con prescripción de abstinencia absoluta, atención mayoritariamente ambulatoria para todo el proceso de tratamiento con terapia individual, farmacoterapia, prevención de recaídas, coordinación con unidades de hospitalización para adquisición de la abstinencia, consolidación de la abstinencia y manejo de complicaciones psiquiátricas y coordinación con grupos de autoayuda ${ }^{(2)}$. Sin embargo, la mayoría de los dependientes del alcohol Sin Hogar, van a necesitar una adaptación de este programa a sus características, sobre todo, para paliar las altas tasas de abandono y las dificultades en el inicio y mantenimiento de la abstinencia. En este sentido, los objetivos y las estrategias van a ser los de los programas de reducción de daños ${ }^{(42)}$ : priorización de la retención en el tratamiento, intervenciones sobre las complicaciones del consumo y cambio de prescripción, desde la abstinencia absoluta a la reducción de los niveles de consumo.

\section{Adherencia al tratamiento}

La retención en el tratamiento se va a convertir en un objetivo primordial y en indicador de calidad del programa. Los buenos resultados se asocian, como ocurre en otras adicciones, a: fácil acceso al tratamiento, accesibilidad física del centro y horarios adecuados, accesibilidad de los miembros del equipo, calidez y permanencia del personal, coordinación con servicios sociales, diversidad de los servicios ofrecidos, orientación del tratamiento hacia el largo plazo, tratamiento focalizado en la rehabilitación, actitud del equipo terapéutico caracterizado por no presionar a los pacientes hacia la abstinencia, planteamiento del programa a plazo indefinido y flexibilidad e individualización del marco terapéutico ${ }^{(43)}$.

Intervención sobre las complicaciones del consumo

El alivio o resolución de las complicaciones somáticas va a mejorar la calidad de vida de los pacientes y va actuar como factor reforzante de la disminución del consumo. Su manejo implica la coordinación con Atención Primaria y con las Unidades de Hospitalización correspondientes. Lo mismo ocurre con los trastornos psiquiátricos asociados, cuya persistencia y manejo van a estar directamente relacionados con el control del consumo, y cuyo abordaje, en la mayoría de las ocasiones, no se va a poder hacer a nivel ambulatorio. La hospitalización psiquiátrica, ya sea en unidades generales, de patología dual o específicas para problemas de alcohol; seguirá siendo un recurso imprescindible tanto para resolver situaciones de crisis, violencia o riesgo autolítico, como para desintoxicar y controlar el síndrome de abstinencia. El establecer periodos continuados de abstinencia que permitan el manejo de estas complicaciones, va a requerir prolongar las estancias de internamiento y el uso de hospitalizaciones parciales, hospital de día, durante meses, antes de ser remitidos al programa ambulatorio.

El deterioro psicoorgánico y las demencias son una complicación terminal de muchos pacientes, que han sobrevivido a muchos años de consumo de grandes cantidades de alcohol y déficit nutricionales. Su manejo va a depender de las facilidades de acceso a la red normalizada de unidades residenciales.

\section{Intervenciones sobre el consumo}

La redución de los niveles de consumo va a necesitar de intervenciones psicoterapéuticas y psicofarmacológicas. Las intervenciones psicoterapéuticas que se han mostrado eficaces son aquellas que se llevan a cabo en el contexto de una buena relación terapéutica y con un estilo empático, reflexivo y cálido; informan al paciente acerca de sus condiciones físicas, psicológicas y sociales y de los efectos y consecuencias del abuso de alcohol; le transmiten que es dueño y responsable de sus actos y que tiene capacidad para elegir y tomar la decisión que quiera con respecto a cambiar su conducta de bebida; le aconsejan claramente sobre la necesidad de cambiar; le ofrecen distintas alternativas para hacerlo; Ilegan a un acuerdo concreto de los objetivos a conseguir y refuerzan la confianza del paciente en si mismo y en sus expectativas de que realmente puede lograr los objetivos propuestos ${ }^{(45)}$. 
Fijar los objetivos de bebida es un factor fundamental. Tras establecer la línea base de consumo en los últimos seis meses, se puede iniciar un objetivo de reducción de una cuarta parte de su consumo actual a lograr en el proximo mes y mantener ese nivel un mes más. Una vez conseguido el objetivo, se marcarán nuevos objetivos siguiendo la misma norma ${ }^{(46)}$

Desde el punto de vista farmacológico, clásicamente, se han utilizado las benzodiacepinas de vida media media o larga y otros sedantes, cuyo uso de forma prolongada ayudaba a reducir los niveles de consumo; pero las interacciones con el alcohol, la presencia de efectos paradójicos y el riesgo de escalada de dosis y dependencia han favorecido la búsqueda de fármacos alternativos. Los medicamentos mas útiles son aquellos sin capacidad adictiva y sin interacciones graves con el alcohol. La lista es muy amplia: Fármacos serotoninérgicos (citalopram, fluvoxamina, fluoxetina, sertralina, zimelidina, buspirona, ondansetron y ritanserina); antagonistas opioides (naltrexona y nalmefene); fármacos dopaminérgicos (bromocriptina, apomorfina, tiapride y zuclopentixol); agonistas gabaérgicos (acamprosato y gamma hidroxibutírico) y estabilizadores (litio, gabapentina, topiramato). De todos ellos, los inhibidores selectivos de la recaptación de la serotonina, el acamprosato y la naltrexona son los que se han mostrado más útiles en la práctica clínica, sobre todo los dos últimos ${ }^{(47)}$. Se debe mantener el tratamiento durante periodos muy prolongados $y$, en la elección del fármaco, tener en cuenta la presencia de patología orgánica asociada y las interacciones con otras medicaciones.

En aquellos casos con dependencia de alcohol muy severa, con incapacidad para mantener la abstinencia o la reducción significativa de los niveles de consumo, puede ser necesario plantearse el tratamiento en regimen de internamiento prolongado. La utilidad de las Comunidades Terapéuticas va a depender de la adaptación de las mismas a las características de estos pacientes ${ }^{(48)}$ : con un compromiso bajo, alta comobilidad médica y psiquiátrica y objetivos de reducción de consumo. Las unidades específicas de media y larga estancia, más de seis meses, puede ser una opción válida; aunque la baja tasa de retención y los cortos periodos de permanencia en las mismas, suele hacer necesario su uso de forma frecuente y reiterada.

En aquellos dependientes del alcohol Sin Hogar que no acuden a los Servicios de Salud Mental, los programas de reducción de daños, con ingredientes similares a los descritos en los programas de los Servicios de Salud Mental, se pueden llevar a cabo en el medio en el que se muevan, a través de los equipos específicos de calle (Equipo Multidisciplinar de Enganche y Tratamiento de Enfermos Mentales Sin Hogar).

\section{REQUISITOS PARA LA IMPLANTACION DE PRO- GRAMAS INTEGRADOS}

Para la implantación de programas específicos integrados en los dispositivos generales, se va a precisar de la daptación, tanto de los dispositivos generales como de los programas específicos para problemas de alcohol.

El funcionamiento y eficiencia de estos programas, descansa en los siguientes ejes ${ }^{(6)}$ : 1) Coordinación de los servicios que intervienen, fundamentalmente servicios sociales generales y específicos y servicios sanitarios y de salud mental. 2) Discriminación positiva de estos usuarios en todos los recursos de la red, con amplia disponibilidad y adaptación de los programas a sus necesidades. Garantía de camas hospitalarias para situaciones urgentes, adaptación de criterios de ingreso, estancias medias y coordinación de altas con los servicios ambulatorios. 3) Creación de equipos multidisciplinares específicos, insertados en la red de salud mental de las áreas implicadas, pero con movilidad y trabajo en la calle, y con una dependencia mixta de Salud Mental y Servicios Sociales. 4) Creación de recursos de alojamiento con distintos niveles de exigencia: desde centros de atención permanente a Enfermos Mentales Sin Hogar, ubicados en las areas donde están esto pacientes, que proporcionan un lugar de estancia (24 horas/365 días), satisfacen las necesidades básicas, abordan las necesidades especializadas de atención, y facilitan el acceso a otros servicios de la comunidad; hasta Centros de Acogida con diferentes niveles de exigencia, que deben ser puntos de enganche con capacidad de detección de casos y derivación a los equipos de intervención. 5) Creación de ayudas económicas para cubrir las necesidades básicas (pensiones no contributivas, ingreso de integración, ingreso de rentas mínimas, etc.).

Los programas para problemas de alcohol, de los servicios de salud mental o de los servicios de drogodependencias, necesitarán adaptar sus objetivos y estrategias de intervención, no solo para poder cubrir la amplia gama de necesidades que estos pacientes tienen sino para facilitar el enganche, manejar la dependencia de alcohol con objetivos flexibles con respecto al consumo, y manejar la patología psiquiátrica asociada, presente en casi todos los casos. Con este fin, se hacen imprescindibles: equipos multidisciplinares (psiquiatras, psicólogos, diplomados en enfermería y trabajadores sociales), formación del equipo en el manejo de trastornos psiquiátricos en general y de trastornos por uso de sustancias en particular, y trabajo a través de estrategias de seguimiento de casos individualizado (case management).

\section{REFERENCIAS}

1 Monrás M, García S, Torres M, Gual A, Ortega L. El ingreso hospitalario en el tratamiento de los alcohólicos cronificados. Adicciones 1998; 10: 151-160. 
2 Cuadrado P, García R, Pata MD, Noval MJ. Respuesta de la dependencia alcohólica a un programa comunitario de tratamiento. Psiquiatría Pública 1998; 10: 162-168.

3 Cave P, Martin I, Wilson G. Homeless drinkers, in T Lawrence (Comp.) Teaching about alcohol problems. Tutors Manual and Student hand-outs. Cambridge, Woodhead-Faulkner Limited 1987: 74-82.

4 Koegel P, Burnam A.. Alcoholism among homeless adults in the inner city of Los Angeles. Arch Gen Psychiatry 1988; 45: 1011-1018.

5 Muñoz M, Vazquez C, Vazquez JJ. Atrapados en la calle. Madrid, Consejería de Sanidad y Servicios Sociales, Comunidad de Madrid 1998.

6 Grupo SMES MADRID. Propuesta de actuación coordinada en la atención a personas sin hogar con trastornos mentales. Boletín de la Asociación Madrileña de Salud Mental (AEN) 2001, primavera: 5 -15.

7 Drake M. Homeless people in Europe and their rights. Tercer Informe del Observatotio Europeo de los Sin Techo. Bruseles, FEANTSA 1994.

8 Arce AA, Vergara MV. Identifying and characteristics the mentally ill among the homeless, in Lamb HR (ed.): The Homeless Mentally III. Washington, DC, American Psychiatric Press INC.1984.

9 Fischer PJ, Breakey WR. Homelessness and mental health: an overview. Int. J. Mental Health 1986, 14: 641.

10 Gelberg L, Linn LS, Leake BD. Mental health, alcohol and drug use, and criminal history among homeless adults.

Am J Psychiatry 1988, 145: 191-196.

11 Morrisey JP, Dennis DL. Homeless and mental illness: Toward the Next Generation of Research Studies, New York, Office of Programs for the Homesless Mentally III, National Institute of Mental Health 1990.

12 Scott J. Homelessness and Mental Illness. Br J Psychiatry 1993, 162: 314 - 324.

13 Bassuk EL, Rubin L, Laiviat A.. In homelessness a mental health problem? Am J Psychiatry 1984, 141: 1546.

14 Segal B. Homelessness and drinking. A study of a street population. New York, Haworth Press 1991.

15 Mcnamara C, Schumacker JE, Milby JB, Wallace D, Uslan $S$. Prevalence of nonpsychotic mental disorders does not affect treatment outcome in a homeless cocaine dependent sample. Am J Drug Alcohol Abuse 2001, 27: 91- 106.

16 Kasprow WJ, Rosenheck R. Substance use and psychiatric problems of homeless native american veterans. Psychiatric Services 1998, 49: 345 -350.

17 Koegel P, Burnam A, Farr RK. The prevalence of specific psychiatric disorders among homeless individuals in the inner City of Los Angeles. Arch Gen Psychiatry 1988, 45: 1085 - 1092.

18 Susser E, Struening EL, Conorer S. Psychiatric Problems in homeless men. Lifetime psychosis, substance use, and current distress in new arrivals at New York City shelters. Arch Gen Psychiatry 1989, 46: 845 - 850.
19 Vega LS. Salud mental en la población sin hogar. Oviedo, Principado de Asturias: Cuadernos Asturianos de Salud, Consejería de Servicios Sociales 1996.

20 Rico P, Vega SL, Aranguren L. Trastornos psiquiátricos en transeuntes:un estudio epidemiológico en Aranjuez. Revista de la Asociación Española de Neuropsiquiatria 1994, XIV, 51: 633 - 649.

21 Lucas R, Batista G, Borras V, Catarinen S, Sánchez-Salus I, Valls E. Prevalencia de sintomatología psicótica y hábitos tóxicos en una muestra de "homeless". Rev Psiq Fac Med Barna 1995, 22: 18 - 24.

22 Wright JD, Devine JA. Factors that interact with treatment to predict outcomes in substance abuse programs for the homeless. J Addictive Diseases 1995, 14: 169-181.

23 Koegel P, Burnam MA. The epidemiology of alcohol abuse and dependence among homeless individual: Finding from the inner City of Los Angeles. Rockville, $\mathrm{MD}$, National Institute of Alcohol Abuse and Alcoholism 1987.

24 Muñoz M, Koegel P, Vázquez C, Burnam A, San J. Comparison among the patterns of sustance use among the homeless in Madrid (Spain) and Los Angeles (USA). Social Psychiatry and Psychiatric Epidemiology (in press).

25 Castaneda R, Lifshutz H, Galanter M, Franco H. Age at onset of alcoholism as a predictor of homelessness and drinking severity. J Addictive Diseases 1993, 12: 65-77.

26 Alarcon C. Alcoholismo en situaciones de marginación. La actuación de los Serveis Socials de a L'ajuntement de Barcelona en la población de los albergues de Valldocella y San Juan de Dios en el periodo 1980-1982. Libro de Ponencias Socidrogalcohol, XVIII Jornadas Nacionales. Barcelona 1990: 457 - 469.

27 Drake RE, Osher FC, Wallach MA. Homelessness and dual diagnosis. Am Psychology 1991, 46: 1149 - 1158.

28 Uslan SL, Schumacker JE, Milby JB, Wallace D, McNamara C, Michel M. Crack cocaine, alcohol, and other drug use patterns among homeless persons with other mental disorders. Am J Drug Alcohol Abuse 2001, 27: 107-120.

29 Nuttbrock LH, Ng-Mak DS, Rahav M, Rivera JJ. Pre-and post-admission attrition of homeless mentally ill chemical abusers referred to residential programs. Addiction 1997, 92: 1305-1316.

30 Westermeyer J. Nontreatment factor affecting treatment outcome in substance abuse. Am J Drug Alcohol Abuse 1989, 15: 13 - 29.

31 Feuerlein W. Treatment of particular populations of alcoholics. Alcología 1993, 5: 153 - 158.

32 Galanter M, Egelko S, Edwards H, Vergaray M. A treatmen system for combined psychiatric and addictive illness. Addiction 1994, 89: 1227-1235.

33 Drake RE, Essock SM; Shaner A, Carey KB, Minkoff K, Kola L, Lynde D, Osher FC, Clark RE, Richards L.. Implementing dual diagnosis services for clients with severe mental illness. Psychiatric Services 2001, 52: $469-476$. 
34 Bennet MI y col. The value of Hospital Based Treatment for the Homeless Mentally III. Am J Psychiatry 1988, 145: $1273-1276$.

35 Drake RE, Bartels SJ, Teague GB, Noordsy DL, Clark RE. Treatment of substance abuse in severelly ill patients. J Nerv Ment Dis 1993, 181: 606 - 611.

36 Wittman FD, Madden PA. Alcohol Recovery for Homeless People: A survey of current programs in the U.S. Rockville (Maryland), National Institute on Alcohol Abuse and Alcoholism 1988.

37 Torrey EF. Continous treatment teams in the care of the chronic mentally ill. Hosp Community Psychiatry 1986, 37:1243 - 1247.

38 Drake RE, Yorevitch NA, Bebout RR, Harris M, McHugo GJ.Integrated treatment for dully diagnosed homeless adults. J Nerv Ment Dis 1997, 185: 298 - 305.

39 Blankertz LE, Cnaan RA. Assessing the impact of two residential programs for dually diagnosed homeless individuals. Soc Serv Rev 1994, 68: 536 - 560.

40 Mansilla F, Vega P, Gómez-Racevedo MM, Márquez P. Drogodependientes sin hogar: una experiencia. Revista Española de Drogodependencias 1997, 22: 105-113.

41 Martín E. El futuro de los usos de drogas: la estrategia nacional 2000-2008. Trastornos Adictivos, 2001, 3: 15-17.
42 Fernández Liria A, García MI. Los programas de case management, conceptos básicos y aplicabilidad. Revista de la Asociación Española de Neuropsiquiatria 1990, 32: 65-75.

43 Cuadrado P. ¿Son necesarios los programas de reducción de daños en el tratamiento de la dependencia alcohólica? Adicciones 2000, 12: 207 - 214.

44 Del Río M. Farmacoterapia de sustitución en la dependencia a opiaceos. Monografías en Psiquiatría 1998; X: 3-12.

45 Bien TH, Miller WR, Tonigan JS. Brief interventions for alcohol problems: a review. Addiction 1993; 88: 315336.

46 Cuadrado P. Programas de reducción de daños y riesgos en el alcoholismo, en Rubio G y Santo-Domingo J (Coord.) Curso de especialización en alcoholismo. Madrid, Fundación de Ayuda contra la Drogadicción 2001, cap. 10.

47 Soyka M. Profilaxis de las recaídas en el alcoholismo. Avances recientes y posibilidades futuras. CNS Drugs 1997, 7: 313 - 327.

48 Llorente JM, Fernández C, Gutiérrez M. La Comunidad terapéutica en el tratamiento del abuso y dependencia de sustancias. Monografías en Psiquiatría 1998, X: 43-52. 